\title{
A travel clinic's role in reducing measles and pertussis in the community
}

\author{
Alwyn Rapose* \\ Division of Infectious Diseases, Reliant Medical Group,123 Summer Street, Worcester, MA. 01608, USA
}

\begin{abstract}
Background: There were an increased number of cases of measles in the United States in the year 2011, and in 2012 the US recorded the most number of pertussis cases in a single year compared to the previous fifty years. A Travel Clinic can serve as an important contact point to increase uptake of vaccines against these two diseases.

Methods: There was an increased emphasis on testing for measles immunity and vaccination with MMR and Tdap vaccines in persons seeking pre-travel counseling at our Travel Clinic in the year 2011. A retrospective chart analysis of patients seen at the Travel Clinic was performed in 2012. We evaluated the number of MMR and Tdap vaccines administered, as well as the number of persons tested for measles immunity by serology.

Results: 1261 persons were evaluated for in-person consultation in 2011. In addition to traditional "travel vaccines", 133 MMR vaccines and 397 Tdap vaccines were administered. In addition, 416 patients were evaluated for measles immunity by serology testing.

Conclusions: The Travel Clinic is accessed by a large number of persons prior to their travel abroad. Those who are not up-to-date with Tdap vaccine and those not immune to measles have an opportunity to receive the recommended vaccines at their pre-travel visit. The Travel Clinic hence represents a unique opportunity to increase the uptake of MMR and Tdap vaccines in healthy individuals who may not otherwise visit their primary-care-providers, thus reducing the overall risk of outbreaks in the community.
\end{abstract}

\section{Introduction}

Measles transmission was "effectively eliminated" in the United States and Canada around 1998 [1] and post-elimination surveillance was elegantly reviewed by Fiebelkorn Parker and colleagues [2]. However, the Centers for Disease Control and Prevention (CDC) reported 222 cases of measles and 17 outbreaks in the United States in 2011 [3]. On the other hand, pertussis is considered endemic in the United States with frequent small outbreaks. Unfortunately, there were an increased number of pertussis cases, including a large outbreak in Washington State [4] and CDC officials reported that the United States recorded the most cases $(48,277)$ in 2012 compared to the previous fifty years [5]. The solution to these outbreaks of vaccine-preventablediseases is to improve the uptake of the measles, mumps and rubella (MMR) and tetanus, diphtheria and acellular pertussis (Tdap) vaccines. Unfortunately, there are many practical obstacles to vaccination in adults. A unique opportunity presents itself when otherwise healthy persons make plans to travel out of the country. Travelers are already primed to receive traditional "travel vaccines" against diseases like hepatitis A, typhoid, yellow fever, Japanese encephalitis and others. Their visit to a travel clinic (TC) presents an opportunity to review their immunization status and offer the MMR and Tdap vaccines. In this study, the number of patients who received the MMR and Tdap vaccines in our TC during the year 2011 is reported. In addition, the number of patients tested for measles immunity is also reported.

\section{Methods}

The Reliant Medical Group (RMG) is a private multi-specialty group serving the community in Central Massachusetts. RMG utilizes an electronic medical records (EMR) system. The Division of Infectious Diseases provides a specialized service for patients traveling abroad. This is the Travel Clinic. In 2011 the clinic was staffed by four providers - three ID certified physicians (MD) and one nurse practitioner (NP). Vaccines were administered by three certified nurses. In-person evaluation and counseling was provided at patient visits. Telephone consults were also offered for patients who had been previously evaluated at the clinic prior to a previous trip abroad. The majority of patients were referred by community physicians. Self-referred individuals and families formed a smaller percentage of the patients seen. Traditional travel vaccines against hepatitis A, typhoid, polio, meningitis, yellow fever, Japanese encephalitis and rabies were offered. Routine vaccines - hepatitis B, varicella, influenza, pneumococcus, MMR, Tdap and Td were also available. Counseling regarding diarrheal disease, mosquito and other insect-borne-illnesses like malaria, dengue fever and other travel-related issues was an integral part of the consult. At the visit, the patient's EMR was reviewed for allergies, recent medications and previous vaccinations. The CDC's travel information for the patients' respective country of travel helped guide final vaccination recommendations.

Correspondence to: Alwyn Rapose, Division of Infectious Diseases, Reliant Medical Group,123 Summer Street, Worcester, MA. 01608, USA, Tel: (508) 3683122; E-mail: alwyn.rapose@reliantmedicalgroup.org

Keywords: measles, pertussis, travel vaccines, infectious diseases

Received: September 17, 2016; Accepted: September 26, 2016; Published: September 28, 2016 
In response to the reported increased measles and pertussis outbreaks in 2011 our TC placed added emphasis on measles serotesting and vaccination, along with increased emphasis on Tdap vaccination. Patients whose records did not document 2 previous doses of MMR or previous positive serology for measles were offered serology testing and they were offered MMR vaccine if serology was negative. Similarly, those without documentation of Tdap within the last 10 years were offered the Tdap vaccine. The following year i.e. 2012, EMRs of travelers seen in the TC in 2011 were retrospectively evaluated. Billing codes for MMR and Tdap vaccines and codes for measles serology testing were analyzed. The study was approved by the clinic's institutional review board.

\section{Results}

A total of 1261 patients received in-person consults at the RMG's Travel Clinic in 2011. In addition, telephone consults were also provided during this period. The majority of persons were travelling on holiday to their country of origin. Other reasons for travel included tourism and business, international studies, volunteer missions and pilgrimages. 58\% of these visits occurred in the first six months of the year. The month of June saw the most number of travelers $173(13.7 \%$ of the total) in contrast to August when only 60 (4.7\%) travelers were seen. Traditional "travel vaccines" included typhoid (993), hepatitis A (547), polio (287) and yellow fever (273). Specific to the present study, a total of 416 patients were tested for measles by serology. Vaccine was offered to those who lacked immunity. 133 MMR vaccines were administered during the study period. 397 travelers received the Tdap vaccine. The breakup of the number of patients evaluated, tested for measles immunity, and vaccinated with MMR and Tdap by each of the four practitioners is charted in Table 1.

\section{Discussion}

In the last few years there have been an increased number of cases of measles, and outbreaks of pertussis in Europe and North America. In 2011, 28,000 cases of measles were reported in Europe. France alone had more than 10,000 cases. In North America, Canada reported 700 cases. In the United States, early reports of measles outbreaks were considered "import-associated" (cases of patients returning from travel abroad and their contacts who developed measles) the majority of whom were not vaccinated [3]. Evaluating and containing the spread of these infections results in considerable costs. Coleman et al., [6] estimated a total cost of $\$ 25,000$ to conduct a response to a single imported case of measles. In reality, costs are likely even higher - $\$ 799,136$ - as reported from a measles outbreak in Arizona [7]. The state of Massachusetts also reported an increased number of measles cases in 2011. Most of these cases had history of not being vaccinated or "unknown status." In response, the department of public health published recommendations to ensure two-dose MMR vaccine in children, measles immunity in health-care personnel, along with a new recommendation to ensure measles immunity in international travelers.

Table 1. The breakup of the number of patients evaluated, tested and vaccinated by each practitioner.

\begin{tabular}{|l|c|c|c|c|c|}
\hline & $\begin{array}{c}\text { MD 1 } \\
(\mathbf{n = 2 3 4})\end{array}$ & $\begin{array}{c}\text { MD 2 } \\
(\mathbf{n = 2 2 3})\end{array}$ & $\begin{array}{c}\text { MD 3 } \\
(\mathbf{n = 4 5 9})\end{array}$ & $\begin{array}{c}\text { NP } \\
(\mathbf{n = 3 4 5})\end{array}$ & $\begin{array}{c}\text { Total } \\
(\mathbf{n = 1 2 6 1 )}\end{array}$ \\
\hline Measles serology & 44 & 119 & 216 & 37 & 416 \\
\hline MMR vaccine & 14 & 43 & 63 & 13 & 133 \\
\hline $\begin{array}{l}\text { Tdap } \\
\text { vaccine }\end{array}$ & 56 & 78 & 197 & 66 & 397 \\
\hline
\end{tabular}

$\mathrm{n}=$ number of travelers seen in 2011 .
Pertussis on the other hand, is considered endemic in the United. However, in 2012, there were an increased number of cases, including a large outbreak in Washington State which was labeled an epidemic [4)]. Many years prior, in 2005, the Advisory Committee on Immunization Practices (ACIP) had recommended use of the Tdap vaccine in adolescents and adults up to age 64 years (8). In October 2010, recommendations were expanded to include select patients above age 64 years, and in February 2012 the ACIP recommended use of Tdap in all patients 65 years and older [9]. Unfortunately, in spite of these recommendations, the number of pertussis cases in the United States continued to increase. An analysis of this upswing was published in the New England Journal of Medicine [10].

Vaccination with a single dose of the Tdap vaccine and two doses of the MMR vaccine is the best way to protect individuals against pertussis and measles respectively. Vaccination in adults also helps prevent spread of these infections to infants who are too young to receive these vaccines [11]. Unfortunately, there are practical obstacles to vaccination in adults. Misconceptions about the relationship of vaccines to neurologic diseases persist in some communities. In addition, Tdap is not covered by many state vaccine programs and changes to Medicare Part D have created some difficulty for the smooth administration of vaccines. It is also difficult to capture healthy adult individuals who may not visit their physicians on a regular basis and do not feel the need for vaccines [12]. Challenges of implementation of vaccine guidelines, including safety concerns and public misconceptions along with some recommendations for ways to increase immunization coverage were discussed by Lawrence Hammer and colleagues [13].

As seen by the data in this study, our TC serves a large number of travelers. In a one-year period, in addition to telephone consults, 1261 travelers had face-to-face evaluations with a travel specialist. This compares favorably with the number of travelers seen over a two-year period by Global TravEpiNet a large consortium of 18 travel clinics in the US, where the median number of travelers per site was 201 [14]. Review of the data from our TC for the year 2011 indicated that a high number of persons in the community were not updated to Tdap and MMR vaccines. 397 Tdap vaccines were administered which implied indirect evidence that a considerable number of "healthy persons" in the community were at risk of acquiring pertussis either during their travel abroad or on their return home. Similarly, review of patients' records prior to their travel indicated lack of documented protection to measles in a large number of travelers, once again indicating an "at-risk" population in the community. This resulted in measles serotesting in 416 travelers and MMR vaccination in 133 individuals. These two vaccines - Tdap and MMR- are not considered traditional travel vaccines. However, the TC represented an important contact point to achieve improved vaccination and hence prevent the spread of these two vaccine-preventable-infectious diseases.

Our study has a few limitations. The TC provided telephone consults in addition to face-to-face evaluations and 1261 only represented the number of travelers who were actually seen by one of the four providers in the TC. Hence the total number of patients counseled prior to travel was underestimated. Also, we were unable to capture data on patients who were offered but declined sero-testing and / or vaccination. There was also considerable difference between the four providers regarding the number of travelers seen, as well as the number of serology testing requested and vaccinations ordered. The reasons for these differences were not evaluated. Data regarding MMR and Tdap vaccination during the previous year was also not analyzed. These limitations do not reduce the important findings of 
this observational study. This study reveals a significant gap between MMR and Tdap vaccine recommendations and the implementation of these recommendations. Addressing this gap should involve increasing awareness among physicians and patients of the ongoing epidemics. Resources should be directed towards improving the uptake of these established vaccines, emphasizing vaccination at all physician visits primary care as well as specialty services including TCs.

In conclusion - measles and pertussis - diseases against which there are effective vaccines are re-emerging with dramatic effects as communities are lowering their guard.

However, vaccination with the MMR and Tdap vaccines at a Travel Clinic represents a significant opportunity to interrupt the spread of these diseases.

\section{References}

1. Sever AE, Rainey JJ, Zell ER, Hennessey K, Uzicanin A, et al. (2011) Measles elimination in the Americas: a comparison between countries with a one-dose and twodose routine vaccination schedule. J Infect Dis 204 Suppl 2: S748-755.[Crossref]

2. Fiebelkorn AP, Redd SB, Gallagher K, Rota PA, Rota J, et al. (2010) Measles in the United States during the postelimination era. J Infect Dis 202: 1520-1528.[Crossref]

3. Measles (2016) Morbidity and Mortality Weekly Report (MMWR). CDC.

4. Centers for Disease Control and Prevention (CDC) (2012) Pertussis epidemic-Washington, 2012. MMWR Morb Mortal Wkly Rep 61: 517-522.[Crossref]

5. Pertussis (Whooping Cough) (2015) Pertussis Outbreak Trends. CDC.
6. Coleman MS, Garbat-Welch L, Burke H, Weinberg M, Humbaugh K, et al. (2012) Direct costs of a single case of refugee-imported measles in Kentucky. Vaccine30: 317 321.[Crossref]

7. Chen SY, Anderson S, Kutty PK, Lugo F, McDonald M, et al. (2011) Health careassociated measles outbreak in the United States after an importation: challenges and economic impact. $J$ Infect Dis 203: 1517-1525.[Crossref]

8. Broder KR, Cortese MM, Iskander JK (2006) Preventing tetanus, diphtheria, and pertussis among adolescents: use of tetanus toxoid, reduced diphtheria toxoid and acellular pertussis vaccines recommendations of the Advisory Committee on Immunization Practices (ACIP). MMWR Recomm Rep 55(RR-3):1-34.

9. Centers for Disease Control and Prevention (CDC) (2012) Updated recommendation for use of tetanus toxoid, reduced diphtheria toxoid, and acellular pertussis (Tdap) vaccine in adults aged 65 years and older - Advisory Committee on Immunization Practices (ACIP). MMWR Morb Mortal Wkly Rep25:468-70.

10. Cherry JD (2012) Epidemic pertussis in 2012--the resurgence of a vaccine-preventable disease. $N$ Engl J Med 367: 785-787.[Crossref]

11. Healy CM, Rench MA, Baker CJ (2011) Implementation of cocooning against pertussis in a high-risk population. Clin Infect Dis 52: 157-162.[Crossref]

12. Miller BL, Kretsinger K, Euler GL (2011) Barriers to early uptake of tetanus, diphtheria and acellular pertussis vaccine (Tdap) among Adults-United States, 2005-2007. Vaccine 29: 3850-3856.

13. Hammer LD, Curry ES, Harlor AD, Laughlin JJ, Leeds AJ, et al. (2010) Increasing immunization coverage. Pediatrics 125: 1295-1304.[Crossref]

14. LaRocque RC, Rao SR, Lee J (2012) Global TravEpiNet: a national consortium of clinics providing care to international travelers--analysis of demographic characteristics, travel destinations, and pretravel healthcare of high-risk US international travelers, 2009-2011. Clin Infect Dis 54: 455-462.

Copyright: $(2016$ Rapose A. This is an open-access article distributed under the terms of the Creative Commons Attribution License, which permits unrestricted use, distribution, and reproduction in any medium, provided the original author and source are credited. 